\title{
Resultados del Programa de Prevención de Defectos de Tubo Neural en Chile mediante la fortificación de la harina con ácido fólico. Período 2001-2010
}

\author{
JULIO NAZER H. ${ }^{1}$, LUCÍA CIFUENTES O. ${ }^{2}$
}

\section{Effects of wheat flour fortification with folic acid on the prevalence of neural tube defects in Chile}

Background: Congenital malformations (CMF) have an important role in infant mortality. Neural tube defects (NTD) have great relevance from both social and public health points of view. The ECLAMC (Collaborative Latin American Study of Congenital Malformations) maintains in Chile an epidemiological surveillance of CMF prevalence rate at birth since 1969. Aim: To assess the effect of wheat flour folic acid fortification on the prevalence of NTD. Patients and Methods: Only Anencephaly, Spina bifida and Cephalocele were considered as NTD. All children born in the maternities incorporated to ECLAMC between 1969 and 1999 were considered as belonging to the pre folic acid fortification period and those who were born from 2001 to 2010 were considered as belonging to the post fortification period. Results: The NTD prevalence rate at birth in the pre fortification period was 17.03/10,000. In the second period, there were 291,996 births and among them, 280 newborns were affected by a form of NTD (9.59 in 10,000 births). This represents a $44 \%$ decrease $(p<0.01)$. Anencephaly rate fell from $7.16 / 10,000$ to $3.67 / 10,000$, representing a $49 \%$ lower rate $(p<0.01)$. Spina bifida rate decreased from 8.61/10,000 to 4.49/10,000, representing a $48 \%$ lower rate $(p<0.01)$. Cephalocele had a $20 \%$ non-significant reduction. Conclusions: Wheat flour fortification with folic acid reduced by $44 \%$ the prevalence rate of NTD at birth. This means that NTDs were prevented in 185 Chilean newborns each year.

(Rev Med Chile 2013; 141: 751-757).

Key words: Folic acid; Neural tube defects; Newborns.
'Unidad de Neonatología, Maternidad Hospital Clínico Universidad de Chile. Instituto de Ciencias Biomédicas. Facultad de Medicina, Universidad de Chile.

Recibido el 25 de abril de 2012, aceptado el 17 de enero de 2013.

Correspondencia a: Dr. Julio Nazer Herrera Santos Dumont 999, Santiago de Chile. E-mail:jnazer@ redclinicauchile.cl
L as anomalías congénitas tienen un rol importante dentro de las causas de mortalidad infantil. En Latinoamérica existe desde 1967 un programa de vigilancia epidemiológica de estas patologías al que Chile ingresó en 1969: el ECLAMC (Estudio Colaborativo Latinoamericano de Malformaciones Congénitas) cuyo objetivo principal es la de monitorizar las frecuencias de las malformaciones congénitas (MFC) con el fin de pesquisar variaciones de dichas frecuencias y poder hacer prevención primaria de ellas ${ }^{1}$. Partici- pan 114 maternidades distribuidas en 10 países de Sudamérica ${ }^{2}$. El ECLAMC es, a su vez, miembro del Internacional Clearighouse for Birth Defects Monitoring System (ICBDMS, 1991).

A principios de la década 1990-99 se demostró con trabajos de distribución aleatoria, que el uso de multivitamínicos que contuvieran ácido fólico (AF), reducían significativamente el riesgo de tener hijos afectados con defectos de cierre del tubo neural (DTN) ${ }^{3-5}$.

En la actualidad está demostrado que la admi- 
nistración periconcepcional de $0,4 \mathrm{mg}$ (400 ug) diarios de AF puede prevenir la ocurrencia de 50\% de los DTN y hasta 70\% de las recurrencias si se administra dosis 10 veces mayor, $4 \mathrm{mg}$ diarios ${ }^{6,7}$. El cierre del tubo neural se completa a los 28 días de gestación y la mayoría de las mujeres embarazadas consultan después de pasada esa fecha. Por ello algunos países como Estados Unidos de Norteamérica (USA) a partir de 1998 recomiendan la fortificación con AF de los alimentos de consumo masivo como harinas y cereales ${ }^{8}$.

La tasa de prevalencia al nacimiento de los DTN en las maternidades chilenas participantes del ECLAMC en el período 1982-1999 fue de 17,03 por 10.000 nacimientos ${ }^{10}$, superior a la del resto del ECLAMC que fue de 14,88 por 10.000 .

En Chile la harina de trigo, que es una de las principales fuentes de la alimentación, está siendo fortificada con AF, desde enero del año 2000, por disposición del Ministerio de Salud, con 220 ug de ácido fólico por cada $100 \mathrm{~g}$ de harina, asegurando un aporte de alrededor de 400 ug per cápita ${ }^{11}$. La harina fortificada empezó a ser consumida por la población 4 a 6 meses después (una vez acabado los stocks de harina no fortificada de los molinos), por lo que se esperaba que los $\mathrm{RN}$ cuyas madres recibieron los alimentos fortificados, empezaran a nacer en los primeros días del año $2001^{12}$.

Estudios preliminares realizados por el ECLAMC a 20 meses de iniciado el programa de fortificación ${ }^{13}$ mostraron una reducción significativa de las tasas de prevalencia al nacimiento de los DTN en Chile.

Sin embargo, para poder atribuir a la acción del AF la causa real de esta reducción, es necesario conocer cual era la tendencia histórica de estas tasas, tanto en nuestro país como en el resto del mundo. Estudios de tendencia en Europa, EUROCAT Workin Group ${ }^{14,15}$ y USA ${ }^{16}$ mostraban una tendencia a disminuir las tasas.

Es necesario, además, tener en cuenta que el ECLAMC es un estudio de base hospitalaria, donde los hospitales están continuamente entrando al sistema de vigilancia, permanecen activos algunos años y luego se retiran en diferentes períodos. Son pocos los que permanecen por largo tiempo. El Hospital Clínico de la Universidad de Chile es el único que ha colaborado en forma ininterrumpida desde su ingreso hasta la fecha. Ello puede, al analizar largos períodos, alterar los resultados y por ende modificar la tendencia.
Para verificar si en Chile una tendencia preexistente a la disminución de las tasas pudiera influir sobre el resultado real de la acción del ácido fólico, estudiamos las tasas de prevalencia al nacimiento de una serie de 31 años previos a la fortificación y la comparamos con los datos actuales.

\section{Pacientes y Método}

El ECLAMC fue creado, como dijimos, en 1997. En él participan 114 maternidades de 10 países Sudamericanos ${ }^{2}$, entre ellos Chile desde 1969. La lista de los hospitales chilenos participantes de este estudio se muestra en la Tabla 1.

Ingresan al registro todos los recién nacidos (RN) vivos o mortinatos, cuyo peso de nacimiento sea de 500 g o más. Se consideró DTN a todos casos de anencefalia, espina bífida y meningocele vivos o mortinatos, tanto aislados como asociados a otras anomalías. Ellos fueron definidos de acuerdo a las normas del ICBDMS (1991).

El objetivo de este trabajo es conocer la magnitud de la disminución de las tasas de prevalencia al nacimiento de los DTN: anencefalia, espina bífida y cefalocele en Chile, al cumplirse 10 años de la implementación del programa de fortificación de la harina con AF.

Para poder evaluar los resultados, se consideró como período pre fortificación a los nacimientos ocurridos antes del año 2000 y como post-fortificación los nacidos a partir del año 2001. El año 2000 fue considerado como un año de transición, ya que la harina fortificada comenzó a llegar a la población en marzo o abril de ese año y los niños concebidos dentro del programa comenzaron a nacer seguramente a comienzos del 2001. Para este trabajo se consideró como el período prefortificación la muestra publicada de los años 1982 a $1999^{10}$. Como período post-fortificación el período 2001-2010.

Se revisó la base de datos que el ECLAMC dispone en las maternidades chilenas participantes en el proyecto. Ingresaron a la muestra todos los RN vivos y mortinatos de 500 g o más, que nacieron entre enero de 2001 y diciembre de 2010. Se comparó las tasas entre los períodos pre y post fortificación, mediante prueba de $\chi^{2}$. Se estudió la tendencia de las tasas a través del test no paramétrico de tendencia desarrollado por Cusik ${ }^{17}$. 
Resultados del Programa de Prevención de Defectos del Tubo Neural - J. Nazer et al

\section{Resultados}

En el período estudiado ocurrieron 291.996 nacimientos consecutivos en los hospitales chilenos participantes (Tabla 1). De ellos 289.850 fueron recién nacidos vivos (RNV) y 20.146 mortinatos (RNM), lo que representa una mortinatalidad o mortalidad fetal tardía de $(0,73 \%)$.

En la muestra se encontró 280 pacientes portadores de algún DTN (9,63 por 10.000 nacimientos. De ellos 239 (85,4\%) eran RNV y $41(14,6 \%)$ RNM. La tasa de prevalencia de DTN en NV fue 9,7 por $10.000(280 / 289.850)$ y de $1,9 \%(41 / 2.146)$ en mortinatos.

Al comparar las tasas del período pre fortificación (1969-1999) con el período post fortificación (2001-2010) (Tabla 2), podemos observar una reducción significativa del Total de DTN de 17,03 a $9,58\left(\chi^{2}=63,55\right)$, lo que representa una disminución de $43,71 \%$. Si desglosamos por diagnósticos vemos que anencefalia cayó de 7,15 por 10.000 a 3,66 por $10.000\left(\chi^{2}=35,33\right)$, lo que representa una disminución de 48,81\%. Espina bífida, por su parte bajó de 8,60 por 10.000 a 4,48 por 10.000 $\left(\chi^{2}=47,67\right)$ lo que representa una disminución de $47,9 \%$. Cefalocele disminuyó $20,10 \%$, pero no alcanzó significancia $\left(\chi^{2}=1,57, \mathrm{p}<0,05\right)$ (Tabla 2). El total de casos de RN con DTN es inferior a la suma de los casos de anencefalia, espina bífida y cefalocele, pues algunos $\mathrm{RN}$ presentaban simultáneamente dos de estas malformaciones.

La evolución de esta disminución de las tasas de prevalencia del total y de cada una de las anomalías se muestra en la Tabla 3. Podemos observar que ella no es homogénea, ya que a una caída significativa los años 2002 y 2003 le sigue una alza brusca en el 2004 y a partir de ahí tomar una tendencia a disminuir hasta el año 2009.
La evolución de la tasa total de DTN a lo largo de los años se aprecia en la Tabla 4, en ella se observa que las tasas descienden después del año 2000. La gráfica de estas tasas que se expone en la Figura 1, demuestra una curva que no se ajusta a ningún modelo no lineal y tampoco demuestra periodicidades estadísticamente significativas. Se puede apreciar una diferente tendencia entre el año 1982 y 1999 (ascendente) y el período comprendido entre los años 2001 y 2010 en que la tendencia es descendente. En el período1982-1999 el test de Cuzick demuestra una tendencia al aumento que

\section{Tabla 1. Hospitales chilenos participantes en el ECLAMC en el período 2001-2010}

\begin{tabular}{|l|}
\hline Hospital Clínico de la Universidad de Chile \\
Hospital Regional de Valdivia \\
Hospital Las Higueras de Talcahuano \\
Hospital Naval de Valparaíso \\
Sanatorio Alemán de Concepción \\
Hospital Regional de Rancagua \\
Dr. E. Torres de lquique \\
Hospital Base de Linares \\
Hospital Base de Puerto Montt \\
Hospital Sótero del Río \\
Hospital del Salvador \\
Hospital San Borja-Arriarán \\
Hospital San José \\
Hospital de Talca \\
Hospital de Cauquenes \\
Hospital de Curicó \\
\hline
\end{tabular}

Tabla 2. Comparación entre las tasas de Defectos del Tubo Neural del período 1969-1999 y las del período 2001-2010 en hospitales chilenos miembros del ECLAMC. Tasa por 10.000 nacimientos

\begin{tabular}{|lrrrrrr|}
\hline & $\mathbf{1 9 6 9 - 1 9 9 9}$ & Tasa & $\mathbf{2 0 0 1 - 2 0 1 0}$ & Tasa & Disminución & $\boldsymbol{\chi}^{\mathbf{2}}$ \\
\hline Total nacimientos & 434.624 & & 291.996 & & & \\
Total DTN & 740 & 17,02 & 280 & 9,58 & 43,71 & $68,91^{*}$ \\
\hline Anencefalia & 311 & 7,15 & 107 & 3,66 & 48,81 & $37,03^{*}$ \\
Espina bífida & 374 & 8,60 & 131 & 4,48 & 47,91 & $42,46^{*}$ \\
Cefalocele & 91 & 2,09 & 49 & 1,67 & 20,10 & 1,57 n.s. \\
\hline
\end{tabular}

${ }^{*} p<0,0000001$. n.s. No significativo. 
Resultados del Programa de Prevención de Defectos del Tubo Neural - J. Nazer et al

Tabla 3. Evolución de las tasas por año de anencefalia, espina bífida, cefalocele y total de DTN durante el período 2001-2010 en los hospitales chilenos del ECLAMC. Tasas por 10.000 nacimientos*

\begin{tabular}{|rrrrrrrrrr|}
\hline Año & Total RN & Anencef & Tasa & Esp bifif. & Tasa & Cefalocele & Tasa & Total DTN & Tasa \\
\hline 2001 & 58.893 & 24 & 4,1 & 34 & 5,8 & 13 & 2,2 & 69 & 11,72 \\
\hline 2002 & 51.355 & 14 & 2,7 & 21 & 4,1 & 8 & 1,6 & 43 & 8,37 \\
\hline 2003 & 31.682 & 13 & 4,1 & 16 & 5,1 & 2 & 0,6 & 31 & 9,78 \\
\hline 2004 & 29.266 & 19 & 6,5 & 14 & 4,8 & 6 & 2,1 & 39 & 13,33 \\
\hline 2005 & 27.251 & 10 & 3,7 & 13 & 4,8 & 9 & 3,3 & 28 & 10,28 \\
\hline 2006 & 20.412 & 8 & 4,0 & 10 & 4,9 & 4 & 2,0 & 21 & 10,29 \\
\hline 2007 & 19.280 & 3 & 1,6 & 6 & 3,1 & 3 & 1,6 & 12 & 6,22 \\
\hline 2008 & 20.715 & 6 & 2,9 & 6 & 2,9 & 1 & 0,5 & 13 & 6,28 \\
\hline 2009 & 16.252 & 3 & 1,9 & 4 & 2,5 & 2 & 1,2 & 9 & 5,54 \\
\hline 2010 & 16.718 & 7 & 4,9 & 7 & 4,2 & 1 & 0,6 & 15 & 8,97 \\
\hline Total & 291.996 & 107 & 3,7 & 131 & 4,5 & 49 & 1,7 & 280 & 9,58 \\
\hline
\end{tabular}

*El total de casos de RN con DTN es inferior a la suma de los casos de anencefalia, espina bífida y cefalocele, pues algunos RN presentaban simultáneamente dos de estas malformaciones.

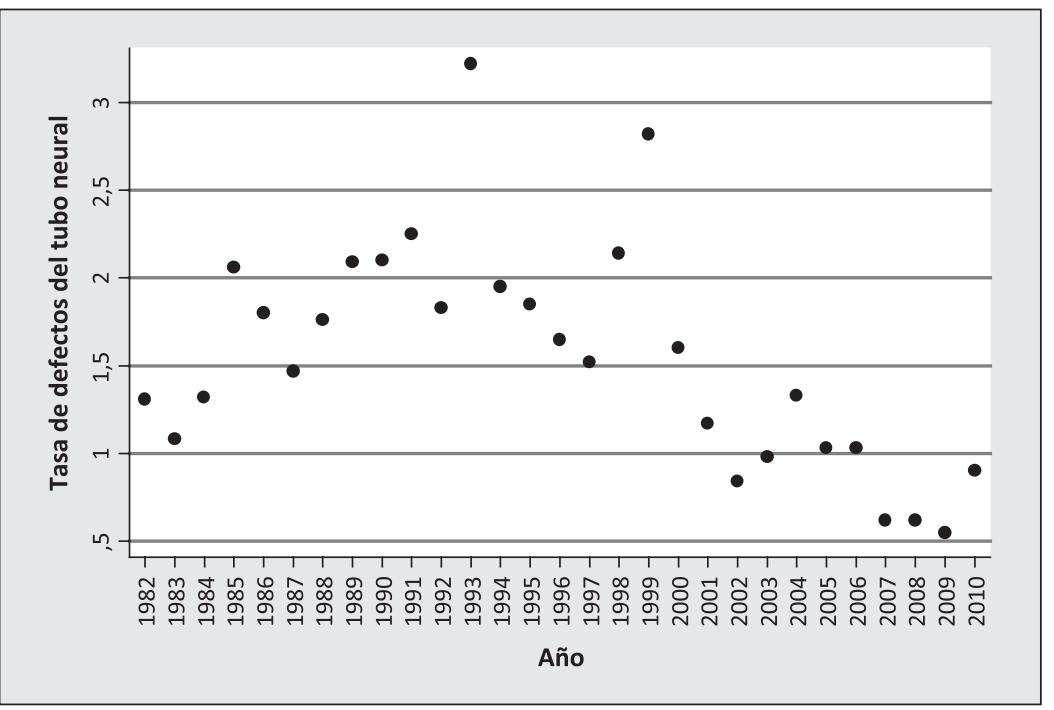

Figura 1. Tasa de defectos tubo neural 1982-2010.

es significativa $(\mathrm{p}=0,023)$ y en el período 2000 y 2010 este test demuestra una tendencia significativa (descenso) en la tasa de defectos del tubo neural entre los años $(\mathrm{p}=0,027)$.

\section{Discusión}

Si estudiamos la tendencia secular de las tasas de prevalencia al nacimiento de los DTN en
Chile en los años previos a la implementación del programa de fortificación de la harina con AF (1967-1999), podemos comprobar que no existía una tendencia a disminuir, si no al contrario a aumentar ${ }^{10}$, como se aprecia en la Figura 1.

La tasa para ese período era de 17,03 por 10.000 nacimientos, significativamente mayor que en el resto del ECLAMC $(14,88$ por 10.000$)$. La reducción de la tasa de prevalencia de 17,03 en el primer período a 9,6 por 10.000 nacimientos en 
Resultados del Programa de Prevención de Defectos del Tubo Neural - J. Nazer et al

Tabla 4. Evolución de las tasas de Defectos de Tubo Neural del período 1969-2010 en hospitales chilenos miembros del ECLAMC. Tasa por 1.000 nacimientos

\begin{tabular}{|l|l|}
\hline Año & Tasa \\
\hline 1982 & 1,31 \\
\hline 1983 & 1,08 \\
\hline 1984 & 1,32 \\
\hline 1985 & 2,06 \\
\hline 1986 & 1,8 \\
\hline 1987 & 1,47 \\
\hline 1988 & 1,76 \\
\hline 1989 & 2,09 \\
\hline 1990 & 2,1 \\
\hline 1991 & 2,25 \\
\hline 1992 & 1,83 \\
\hline 1993 & 3,22 \\
\hline 1994 & 1,95 \\
\hline 1995 & 1,85 \\
\hline 1996 & 1,65 \\
\hline 1997 & 1,52 \\
\hline 1998 & 2,14 \\
\hline 1999 & 2,82 \\
\hline 2000 & 1,6 \\
\hline 2001 & 1,17 \\
\hline 2002 & 0,84 \\
\hline 2003 & 0,98 \\
\hline 2004 & 1,33 \\
\hline 2005 & 1,03 \\
\hline 2006 & 1,03 \\
\hline 2,62 & 0,62 \\
\hline
\end{tabular}

la actualidad es estadísticamente significativa $\left(\chi^{2}\right.$ $63,55)$ y corresponde a una reducción de $42,47 \%$.

Esto demuestra que el Programa de fortificación de la harina de trigo con AF implementada por el Ministerio de Salud de Chile ha tenido un resultado exitoso. La disminución de la tasa de prevalencia al nacimiento de los DTN es muy importante desde el punto vista tanto de salud pública como económico-social. Ello significa que antes del año 2001 con una tasa de 17,03 por 10.000 nacimientos, nacían en Chile 425 niños afectados al año, mientras que en la actualidad con una tasa de 9,58 por 10.000 nacen sólo 240 niños afectados. Se ha logrado evitar que 185 recién nacidos por año presenten esta patología. La anencefalia que es un defecto congénito incompatible con la vida se redujo en casi 50\%, colaborando a la reducción de la mortalidad infantil en forma importante. Lo mismo espina bífida que disminuyó 47,91\%. Se sabe que cerca de $90 \%$ de los niños con espina bífida, si no fallecen en los primeros años, terminan sus vidas en silla de ruedas. La disminución de su prevalencia en casi 50\% constituye un alivio significativo, tanto para la sociedad como para los servicios de salud. La disminución de ambas patologías es estadísticamente significativa, no así cefalocele.

Otro hecho que se demuestra en este trabajo es que las variaciones de las tasas a lo largo de los 10 años han sido muy irregulares, incluso en el año 2004 aumentó por sobre la línea base del año 2001. Luego desde el 2006 se han estabilizado, pero siempre con una leve tendencia a disminuir. Esto sugiere que la carencia de folatos no es el único factor que contribuye a la aparición de estas anomalías y, por lo tanto, es necesario seguir investigando el tema para encontrar otros factores que ayuden a la prevención primaria de ellos.

Si analizamos lo que ha ocurrido en los países de Sudamérica donde se ha implementado programas de fortificación de la dieta con AF, Chile, Argentina y Brasil, podemos observar a través de la literatura médica publicada, la disminución de la frecuencia de los DTN. Castilla y $\operatorname{cols}^{13}$ en el 2003, en una comunicación preliminar, a 20 meses de iniciado el programa en Chile, comunica una reducción de 31\% de los DTN. En un segundo trabajo, con datos definitivos, López-Camelo y cols. $(2005)^{12}$, mostró una reducción de $51 \%$ para espina bífida, con una caída de la tasa desde 9,3 casos por 10.000 nacimientos antes de la fortificación a 4,8 por 10.000 nacimientos después de la fortificación. Una reducción de 43\% en anencefalia, $(8,2 / 10.000$ a 3,2/10.000 nacimientos). La reducción global de los DTN fue de $47 \%$. En otro estudio reciente con datos del ECLAMC, López-Camelo ${ }^{18}$ estudió la frecuencia de 52 defectos congénitos en Chile, Argentina y Brasil y concluye que la fortificación de los alimentos con AF previene los DTN, pero no otros tipos de anomalías congénitas.

Varios países sudamericanos están actualmente 
fortificando la harina de trigo con AF. El primero en hacerlo fue Chile en el año 2000 agregando 2,2 $\mathrm{mg} / \mathrm{kg}$ con lo que proveía 360 microgramos diarios por persona, que es una dosis aceptable para prevenir la ocurrencia de algunos casos de DTN. En el año 2003, 1 año después de iniciado el programa en Chile presentamos los datos preliminares en los cambios en la tasa de prevalencia después de la fortificación de la harina de trigo con $\mathrm{AF}^{13}$, detectados por el método CUSUM, aplicado desde 1999 a todos los países de Sudamérica participantes en el ECLAMC. Se encontró que sólo en Chile los tres tipos de anomalías congénitas de tubo neural, mostraban un descenso significativa (31\%), durante los años 2000-2001 correspondientes al período de fortificación periconcepcional. Los niveles de significancia $(\mathrm{p}<0,001)$ se alcanzaron a los 20 meses después de iniciada la fortificación.

Simultáneamente, en Chile, Eva Hertrampf ${ }^{19}$ comunica que en el período 2001-2002, en una muestra de 120.500 nacimientos, encontró $43 \%$ de reducción en la tasa de DTN. López-Camelo et al. $2005^{12}$, reportan un descenso de la tasa de prevalencia de espina bífida de $51 \%$ y de $42 \%$ en anencefalia en el período post fortificación en Chile.

López-Camelo et al $2010^{18}$ revisan el impacto de la suplementación de la dieta con AF sobre 52 tipos de anomalías congénitas en tres países Sudamericanos con un total de más de 3,3 millones de nacimientos en el período 1982-2007, en Chile, Argentina y Brasil. Comparó los períodos pre y post fortificación y encontró una disminución significativa de la frecuencia de DTN. La máxima reducción de prevalencia se observó en espina bífida aislada, seguida de anencefalia y cefalocele. Sin embargo, estudiados los países por separado se vio que en los tres países hubo un descenso estadísticamente significativo de anencefalia y de espina bífida, pero sólo en Chile y Argentina hubo reducción significativa de las tasas de cefalocele. La reducción de espina bífida fue mayor en los casos aislados que asociados a otras malformaciones congénitas. Es importante destacar que ninguno de los otros 51 diagnósticos estudiados mostraron reducciones significativas.

Carol Bowers ${ }^{19}$, del registro australiano de defectos congénitos, observó en el período 19962006 en que existía ya el programa de fortificación con AF, una disminución de $32 \%$ en la tasa de prevalencia de anencefalia, $23 \%$ en espina bífida y $34 \%$ en cefalocele.
En Canadá, De Wals y cols. ${ }^{20}$, en una muestra de 1,9 millones de nacimientos, durante el período de fortificación, encontraron una disminución de $46 \%$ en la tasa de prevalencia de DTN. Esta disminución fue mayor para espina bífida, $53 \%$, que para anencefalia, $38 \%$, y cefalocele, $31 \%$.

\section{Conclusión}

El programa de prevención primaria de los DTN en Chile mediante la fortificación de la harina de trigo con AF ha logrado en 10 años rebajar la tasa de prevalencia de ellos de 17,02 por 10.000 nacimientos a 9,58 por 10.000 nacimientos, es decir, $43,71 \%$ de reducción. La tasa de anencefalia se redujo de $7,15 / 10.000$ a $3,66 / 10.000$, reducción de 48,81\% ( $\left.\chi^{2} 35,33\right)$. Espina bífida cayó de $8,60 / 10.000$ a $4,48 / 10.000,47,91 \%$ de reducción $\left(\chi^{2} 1,57\right)$. Cefalocele disminuyó $20,10 \%$, no significativo. Esto significa haber evitado que 185 niños al año nacieran con alguna de estas anomalías.

\section{Referencias}

1. Castilla EE, Orioli IM. Epidemiology in neural tube defects in South America. Am J Med Genet 1985; 22 (4): 695-702.

2. Castilla EE, Orioli MI. ECLAMC: The Latin-American Collaborative Study of Congenital Malformations. Community Genet 2004; 7 (2-3): 76-94.

3. MRC Vitamin Study Research Group: Prevention of Neural Tube Defects: results of the Medical Research Council Vitamin Study. Lancet 1991; 338: 131-5.

4. CDC. Use of folic acid for prevention of spina bifida and other tube defects, 1983-1991. MMWR 1991; 40: 513-5.

5. Czeizel AE, Duddas I. Prevention of the first occurrence of neural tube defects by periconceptional vitamin supplementation. N Engl J Med 1992; 327: 1832-5.

6. U.S. Public Health Service: Recommendations for the use of folic acid to reduce the number of cases of spina bifida and other tube defects. MMWR 1992; 41: 1-7.

7. Shaw GM, Schaffer D, Velie EM, Morland K, Harris JA. Periconceptional vitamin use, dietary folate, and the occurrence of neural tube defects in California. Epidemiology 2005; 6: 219-26.

8. Botto LD, Moore CA, Koury MJ, Erickson JD. Neural tube defects. N Engl J Med 1999; 341: 1509-19.

9. Acuña J, Yoon P, Erikson J. La prevención de los defectos de tubo neural con ácido fólico. Estados Unidos. Organización Panamericana de la Salud (OPS), Centro 
para la prevención y el control de Enfermedades (CDC) 1999; 1-13.

10. Nazer J, López-Camelo J, Castilla E. Estudio de 30 años de vigilancia epidemiológica de defectos de tubo neural en Chile y en Latinoamérica. Rev Med Chile 2001; 129: 531-9.

11. Hertrampf E, Cortés F, Erickson JD, Cayazzo M, Freire W, Bailey LB, et al. Consumption of folic acid-fortified bread improves folate status in women of reproductive age in Chile. J Nutr 2003; 133: 3166-9.

12. López-Camelo JS, Orioli IM, Dutra MG, Nazer-Herrera J, Rivera N, Ojeda ME, et al. Reduction of birth prevalence rates of neural tube defects after folic acid fortification in Chile. 2005; Am J Med Genet 135A: 120-5.

13. Castilla EE, Orioli IM, López-Camelo J, Dutra MG, Nazer-Herrera J. Preliminary data on changes in Neural Tube Defects Prevalence rates after folic acid Fortification in South America. 2003: Am J Med Genet 123A: 123-8.

14. EUROCAT Working Group. Prevalence of neural tube defects in 20 regions of Europe and the impact of prenatal diagnosis, 1980-1986. J Epidemiol Commun Health
1991; 45: 52-8.

15. Eurocat Folic acid Working Group. Preventing neural tube defects in Europe: Population based study. BMJ 2005; 330: 574.

16. Elwood JM, Elwood JH. 1980. Epidemiology of anencephalus and spina bifida. Oxford: Oxford University Press.

17. Cuzick J. Wilcoxon prueba tipo para tendencia Stat. Med 1985; 4 (1): 87-90.

18. López-Camelo J, Castilla E, Orioli IM. Folic acid flour fortification: Impact on the frequencies of 52 congenital anomaly types in three South American countries. Am J Of Med Genet 2010; 152 A: 2444-58.

19. Bower C, D'Antoine H, Stanley FJ. Neural tube defects in Australia: trends in encephaloceles and other neural tube defects before and after promotion of folic acid supplementation and voluntary food fortification. Birth Defects Res A Clin Mol Tratol 2009; 85: 269-73.

20. De Wals P, Tairou F, Van Allen MI, Lowry RB, Sibbad B, Evans JA, et al. Reduction in neural-tube defects after folic acid fortification in Canada. N Engl J Med 2007; 357: 135-42. 